\title{
Modelling and Rapid Prototyping of Centrifugal Pump Impeller Based on UG
}

\author{
Yongmei Jiang ${ }^{1}$, Zilong Wang ${ }^{2}$ and Jingnan Zhao ${ }^{3, a}$ \\ ${ }^{1}$ Engineering training center, Harbin University of Science and Technology, Harbin, Heilongjiang, China \\ ${ }^{2}$ Gemini Technologies inc, Egg harbor township, NJ, USA \\ ${ }^{3}$ Rutgers, The State University of New Jersey, Piscataway, NJ, USA
}

\begin{abstract}
The 3D model of centrifugal pump impeller in Unigraphics (UG) was optimized by implementing the principles of NURBS curve interpolation. The impeller was then manufactured by rapid prototyping equipment using polystyrene. The technique improves the efficiency in innovative design, modification, and fabrication.
\end{abstract}

\section{Introduction}

With the development of rapid prototyping, the technique in manufacturing impeller has improved significantly. The 3D model of impeller can be optimized in UG, and the core of impeller can be streamlined after rapid prototyping. Since the core melts into impeller when the temperature reaches certain level during pouring stage, the quality of impeller heavily relies on the 3D modelling. The optimization of impeller requires extensive studies and experiments, as the curve of impeller is determined based upon empirical formula, which may need a series of modifications during modelling and experiment. The modelling of impeller is more effective and accurate by introducing NURBS curve, as the position of individual point, line, and surface can be manually adjusted.

\section{Page layout}

The drawing of impeller includes part drawing and crop drawing. The size of front and rear cover of impeller can be found in part drawing (as shown in Figure 1), while the shape and coordinates of the front and back can be found in crop drawing (as shown in Figure 2). The crop drawing shows the intersections of four cross sections in $10 \mathrm{~mm}$ interval and impeller blade, as well as the points formed by the axial surface every $10^{\circ}$ and blade. Since the curves of impeller can be plotted based on the points on the axis surface with different angles, the crop drawing is the key of generating the curves of impeller [1]. The coordinates of front and rear surface of impeller are shown in Table 1 and 2, respectively. The crop drawing and coordinates are usually provided by the designer for further modelling and fabrication.

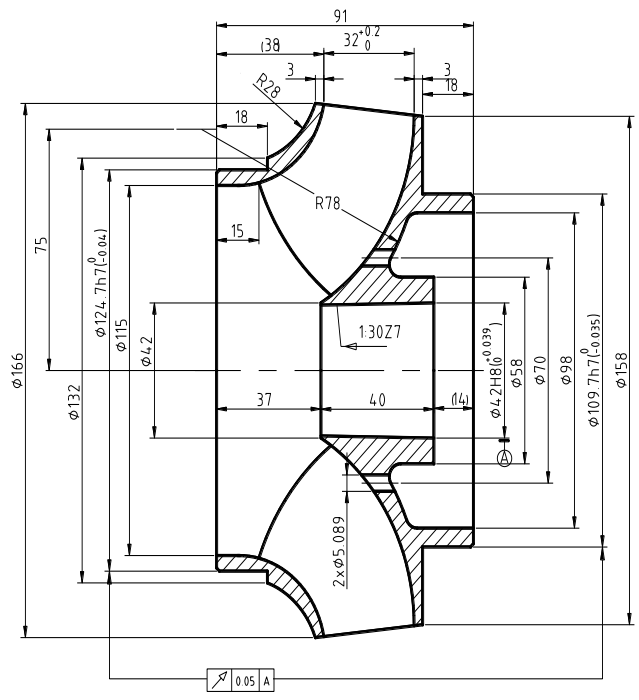

Figure 1. Drawings of part

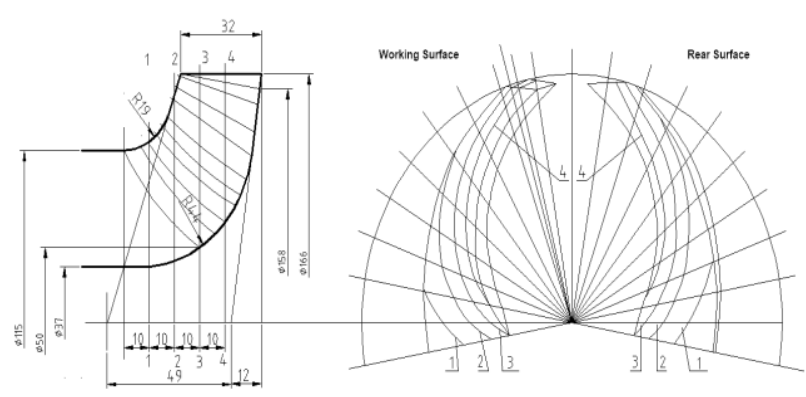

Figure 2. Drawings of corp

\footnotetext{
a Corresponding author: jingnanzhao_cee@yahoo.com
} 
Table 1. Coordinates of impeller (front).

\begin{tabular}{|c|c|c|c|c|c|c|c|c|c|}
\hline \multirow{2}{*}{ Section } & \multicolumn{9}{|c|}{ Degree } \\
\cline { 2 - 10 } & 0 & 10 & 20 & 30 & 40 & 50 & 60 & 70 & 80 \\
\hline $\begin{array}{c}\text { Front } \\
\text { cover }\end{array}$ & 57.5 & 57.8 & 59.5 & 61.5 & 64.5 & 68.3 & 72.8 & 77 & 82 \\
\hline $\mathbf{1 - 1}$ & 43.5 & 51.2 & & & & & & & \\
\hline $\mathbf{2 - 2}$ & 32.5 & 40.5 & 47.5 & 54.5 & 60.5 & 66.5 & 72.2 & & \\
\hline $\mathbf{3 - 3}$ & 25 & 32.3 & 39.3 & 46.6 & 53.3 & 60.1 & 66.8 & 73.2 & 79.6 \\
\hline $\mathbf{4 - 4}$ & & & 33 & 40.4 & 47.5 & 54.5 & 62 & 69.2 & 76.4 \\
\hline $\begin{array}{c}\text { Rear } \\
\text { cover }\end{array}$ & 25 & 28.5 & 33 & 38.2 & 43.8 & 49.5 & 56.8 & 64 & 72 \\
\hline
\end{tabular}

Table 2. Coordinates of impeller (back).

\begin{tabular}{|c|c|c|c|c|c|c|c|c|c|}
\hline \multirow{2}{*}{ Section } & \multicolumn{10}{|c|}{ Degree } \\
\cline { 2 - 11 } & 0 & 10 & 20 & 30 & 40 & 50 & 60 & 70 & 80 \\
\hline $\begin{array}{c}\text { Front } \\
\text { cover }\end{array}$ & 56.5 & 56.8 & 58 & 59.5 & 62 & 65 & 70 & 75 & 81 \\
\hline $\mathbf{1 - 1}$ & 41 & 46 & 52 & 59 & & & & & \\
\hline $\mathbf{2 - 2}$ & 31 & 36 & 41 & 47 & 53 & 60 & 67 & 74 & \\
\hline $\mathbf{3 - 3}$ & 25 & 29 & 34 & 40 & 47 & 53.5 & 61.5 & 69 & 77 \\
\hline $\mathbf{4 - 4}$ & & & & 33 & 40 & 48 & 55 & 63 & 72 \\
\hline $\begin{array}{c}\text { Rear } \\
\text { cover }\end{array}$ & 25 & 26 & 28 & 31 & 37 & 44 & 51 & 59 & 67 \\
\hline
\end{tabular}

\section{Blade surface}

The blade surface is a twisted surface and cannot be interpreted by normal curves or surfaces that formulated by quadratic equation. It can be represented by freeform surface that described by Non-Uniform Rational B-Spline (NURBS) and Implicit algebraic surface. Techniques such as interpolation, regression, approximation are widely used to facilitate the interpretation.

As the spline is constructed from polygons that made up of polylines, it can be generated by approximating the polygons using regression curves. In this case, the shape of spline is determined by the number and vertex of polygon, and the polygon is called the featured polygon or control polygon of the spline.

The $\mathrm{k}$ order of NURBS can be expressed by the rational polynomial vector function shown in Eq.1 [2].

$$
p(u)=\frac{\sum_{i=0}^{n} N_{i, k}(u) \omega_{i} d_{i}}{\sum_{i=0}^{n} N_{i, k}(u) \omega_{i}}
$$

Where $\omega_{i}(i=0,1, \ldots, n)$ is weight factor, and it corresponds to control point $d_{i}(i=0,1, \ldots, n)$. The subscript $\mathrm{i}$ represents serial number, $\mathrm{k}$ is the order. $N_{i . k}(u)$ is the base function of $\mathrm{k}$-th node vector: $U=\left[u_{0}, u_{1}, \ldots u_{n+k+1}\right]$.

The De Boor-Cox's algorithm is used:

$$
\begin{gathered}
N_{i, 0}(u)=1, \quad u_{i} \leq u \leq u_{i+1} \\
N_{i, 0}(u)=0, \text { Others } \\
N_{i, k}=\frac{u-u_{i}}{u_{i+k}-u_{i}} N_{i, k-1}(u)+\frac{u_{i+k+1}-u}{u_{i+k+1}-u_{i+1}} N_{i+1, k-1}(u), k \geq 1 \\
\text { It was assumed that } \frac{0}{0}=0
\end{gathered}
$$

Eq.4 determines the base function of k-th B-Spline. For NURBS, $u_{0}=u_{1}=\ldots=u_{k}, u_{n+1}=\ldots=u_{n+k+1}$ [3].

$R_{i, k}(u)$ in Eq.6 and Eq.7 is the rational base function of NURBS [4]:

$$
\begin{array}{r}
R_{i . k}(u)=\frac{N_{i . k}(u) \omega_{i}}{\sum_{i=0}^{n} N_{i . k}(u) \omega_{i}} \\
p(u)=\sum_{i=0}^{n} R_{i . k}(u) d_{i}
\end{array}
$$

NURBS is widely used in interpreting standard analytical geometry, free curves, and freedom surface. It can change the shape of curve and surface by simply modifying vertex and number of polygons, which is vital in the modeling of curve and surface. UG includes the technique and is effective in generating the $3 \mathrm{D}$ model for impeller.

\section{3D modelling using UG}

To model he hub of impeller, a 2D sketch was first plotted based on the information from part drawing. Then, the 3D model of front and rear covers in impeller hub, as shown in Figure 3, was generated by rotating the sketch by $360^{\circ}$. The model can be further refined by removing certain section using Boolean function. Compared to the blade, the front and rear cover of impeller are easier to model $\lceil 5\rceil$.

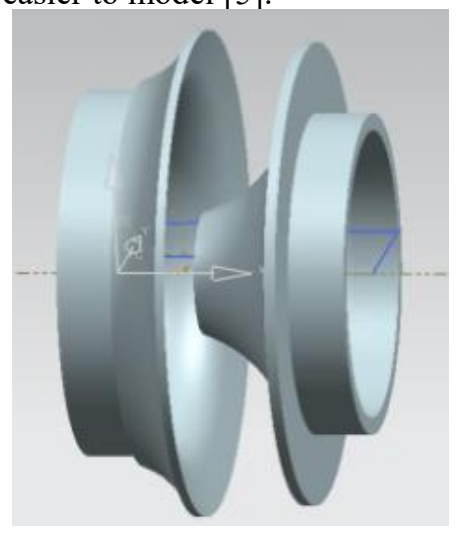

Figure 3. Impeller hub

During the modeling of surface, the point was first created. The curve was generated by connecting the dots, while the surface was created by connecting the curves. There are two methods to construct a curve. In the first method, all the points within the planes under the same vertical axis are connected. In this case, four curves are generated as there are four planes. The surface can be generated by connecting the four curves. The method is not recommended since the generated curves is easily twisted and hard to adjust. In the second method, the sketch is first plotted under certain axis plane. Then, the points that forms the Plane 1, 2, 3, 4, front cover, and rear cover are connected. The generated curves can exclude inflection point by adjusting the smoothness. The method generated a total of nine curves under nine planes ranging 
from $0^{\circ}$ to $80^{\circ}$, and the surface generated by the nine curves are easy to control [6].

\subsection{Generation of points and curves}

A minimum of one author is required for all $\mathrm{B}(25,43.5)$, $\mathrm{C}(35,32.5), \mathrm{D}(45,25)$ were first created in the xy plane in the first sketch. A horizontal line passing $(0,57.5)$ and $(0,25)$ was then created. Point A and E was determined as the intersection of the two horizontal lines and the front and rear cover. Point A, B, C, D ,E were connected in alphabetic order and formed the first curve shown in Figure 4 . The plane every $10^{\circ}$ and the $2 \mathrm{rd}$ to 9 th sketch were created in similar fashion. The intersection between blade, front and rear cover, and plane in each sketch was connected and formed nine curves shown in Figure 5. It is noted that the points should be connected in 2D sketch instead of 3D system, otherwise the curve can be hard to modify [7].

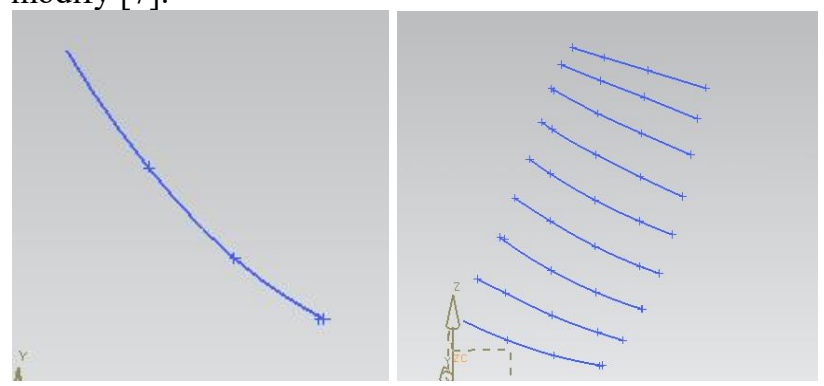

Figure 4. First spline

Figure 5. splines on surface

\subsection{Analysis of curvature comb}

The function "selecting curvature comb" for the first curve in UG is shown in Figure 6. If the curve needs modification, the function should be reselected and cancel previous status. Double click on the curve and adjust the position of control points by dragging the control ball. The smoothness of curve can be adjusted by selecting "editing curve smoothness" and "curvature" in the following drop-down list. The curvature comb of 2 rd to 9 th curves was adjusted to reach ideal situation.

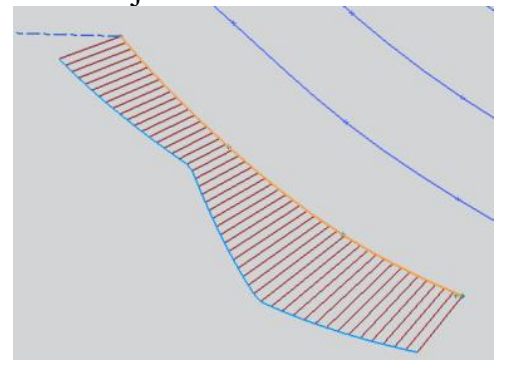

Figure 6. Curvature comb analysis of 1 st spline

\subsection{Generation of surface}

After the curve was calibrated, the surface can be created via "Through curve group". In this case, the nine curves in Figure 5 were connected to form the working surface of the blade shown in Figure 7. The 3D model of the blade can be generated by using function "Thicken" with a thickness of $6 \mathrm{~mm}$, as shown in Figure 8. Based on the coordinates of working surface and nine curves in the same plane, nine curves were generated to form the rear side of the blade. The thickness of inlet, middle, and outlet of the blade is $3 \mathrm{~mm}, 5 \mathrm{~mm}$, and $6 \mathrm{~mm}$, respectively. Figure 9 shows the working and rear surfaces of blade.
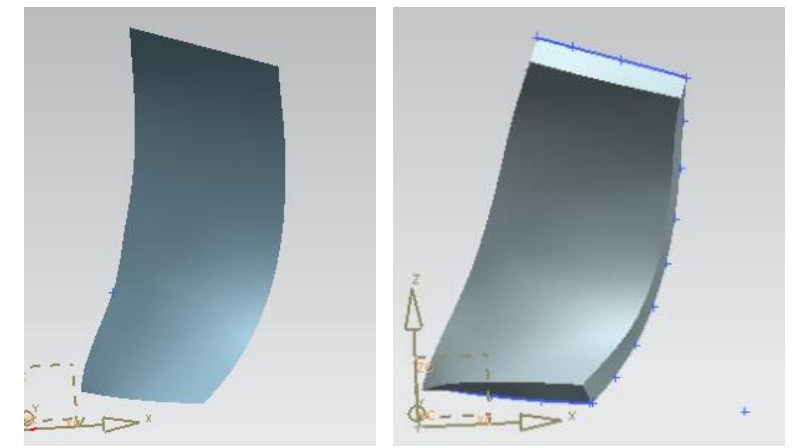

Figure 7. Blade surface Figure 8. Thickened Blade Surface

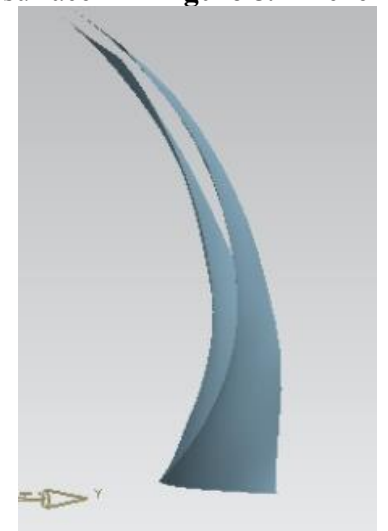

Figure 9. Working and rear surface

\subsection{Analysis and inspection of blade surface}

Due to the accuracy of the coordinates, the curvature may change significantly near the transition zone of the blade surface, leading to a twisted surface. After the generation of surface, the analysis based on light and shadow can overcome the issue by finding and adjusting the curves in the unsmooth area [8]. The function is under "Analyze radius of shape surface- Surface analysis- Radius" in UG. Figure 10 shows the result after selecting the blade and executing the function. Figure 11 is the result after adjusting the reflection. To generate the model, modified blade in Figure 12 was cropped based on the rear surface, and the working surface remained unchanged.

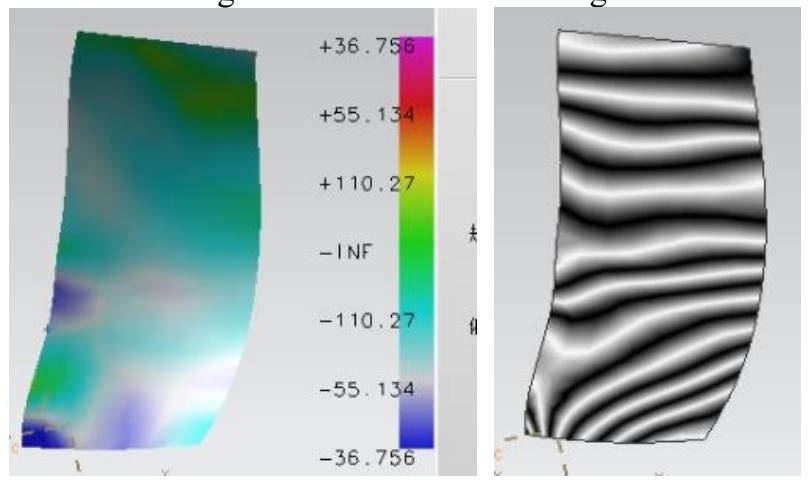

Figure 10. Shape analysis

Figure 11. Light and shadow 


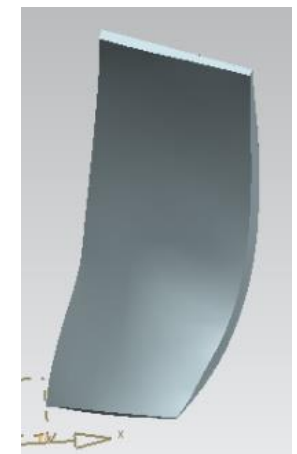

Figure 12. Blade after chopping

\subsection{Modelling of full impeller}

There is gap between the blade and the front and rear cover after the blade was created. Thus, the part of blade near the covers was thickened by $2.5 \mathrm{~mm}$. The other five blades were created by copying and rotating the first blade, as shown in Figure 13. Finally, the impeller was created by applying Boolean function between impeller hub and the blades, as shown in Figure 14.

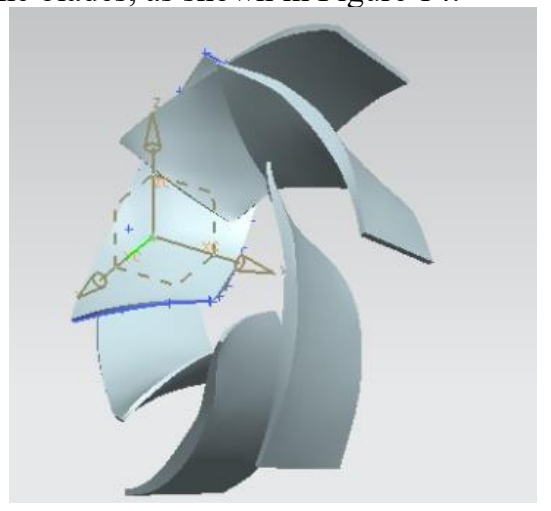

Figure 13. Blade groups

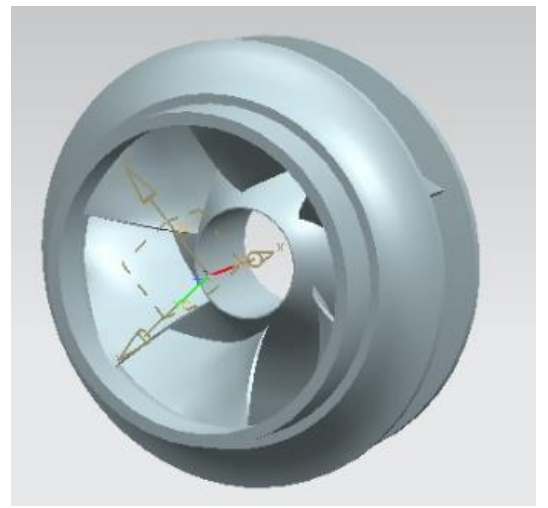

Figure 14. Full impeller model

\section{Rapid prototyping}

The impeller model generated by UG was exported in STL format for rapid prototyping device HRPS-IIIA. The layout of the model was further adjusted by the software. Some parts were enlarged, rotated and merged. During rapid prototyping, polystyrene was added repeatedly. The mold shown in Figure 15 was waxed after prototyping. The mold was identical to the impeller model shown in Figure 16, which suggests that the impeller created by rapid prototyping can be used as the mold for further experiment of new product. The surface of the impeller was coated with several layers of high temperature resistant materials, which also acts as a thin hard shell. During casting, the polystyrene melted rapidly when the molten iron poured in, while the high temperature resistant materials didn't deform and formed the cavity of impeller. The impeller made of metal can be assembled with other parts, such as inlet, and diversion shell pump shaft, to create the main body of water pump. New experiments regarding the efficiency of water pump and the shape of impeller can be conducted nonstop, and any flaw in the impeller can be adjusted easily. Under this circumstance, the design cycle of new product greatly reduced. The complicated impeller can be prototyping without using other cutting tools, molds, or fixtures, which is vital during innovative design. The quality of impeller blade modeling is the key for the whole process.

\section{Conclusion}

The study generated the curves of impeller based on the principles of NURBS curve, interpolation, and approximation. The accuracy and efficiency of the impeller model was guaranteed by the powerful functions related to curve groups and surface in $\mathrm{UG}$, which is beneficial for further $3 \mathrm{D}$ prototyping. The mold was manufactured efficiently using HRPS-IIIA device. The study provides valuable insights for the experiment of new product.

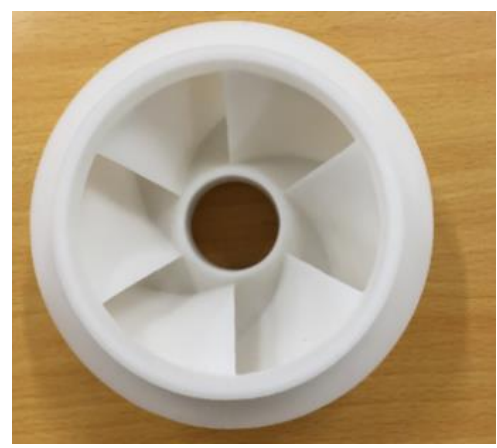

Figure 15. Rapid prototyping of impeller

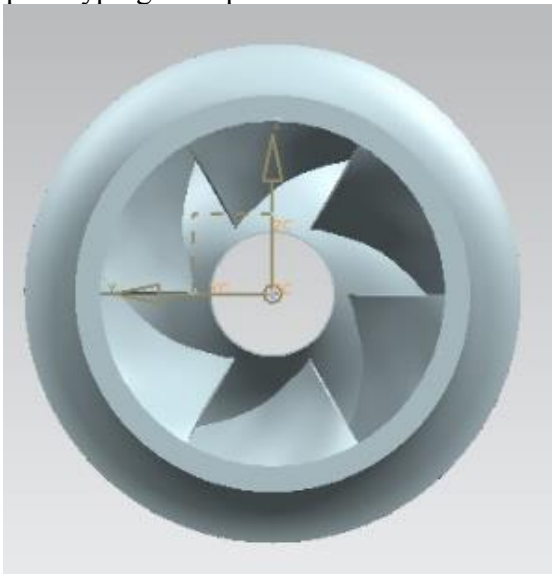

Figure 16. Left view of impeller model 


\section{References}

1. Q. Du, H. Lin, X. Wei, J. Liu. 3D Development of centrifugal pump impeller blade using UGNX. Machine Tool \& Hydraulics, 38, 21 (2010).

2. J. Hu, X. Lv. The Modeling Design of Centrifugal Pump Impeller Based on UG. Journal of Agricultural Mechanization Research, 4 (2011).

3. D. Yu, J. Han, L. Xia. Modeling and Machining Research of Cubic NURBS Spline Curve Interpolation Processing Helical Rotor. Manufacturing Technology \& Machine Tool, 3 (2011)
4. X. Li. Discrete NURBS Surface Interpolation Algorithm Based on Parameters Line Method, Techniques of Automation and Application, 33, 7 (2014).

5. J. Han, K. Li, L. Xia. Machinery, 48, 551 (2010).

6. C. Yan, G. Jia. Modeling of Impeller Based on UGNX4.0. J. Dalian Jiatong Univ., 10 (2009).

7. J. Zhou, W. Zhang. The Research on the Free-form Surface Modeling of Overall Impeller. Mechanical Engineer, 12 (2010).

8. X. Rao, H. Fu, J. Wang. Parametric Modeling for Impeller Blade on UG. Journal of Nanchang Univ. (Engineering \&Technology), 32, 4 (2010). 